\title{
ORDERS AND FAMILIES OF SEED PLANTS OF CHINA
}


This page is intentionally left blank 


\section{ORDERS AND FAMILIES OF SEED PLANTS OF CHINA}

\section{Hsuan Keng}

Department of Botany

National University of Singapore

\section{De-Yuan Hong}

Institute of Botany

Academia Sinica, Beijing

\section{Chia-Jui Chen}

Institute of Botany

Academia Sinica, Beijing 
Published by

World Scientific Publishing Co. Pte. Ltd.

P O Box 128, Farrer Road, Singapore 9128

USA office: Suite 1B, 1060 Main Street, River Edge, NJ 07661

UK office: 73 Lynton Mead, Totteridge, London N20 8DH

\section{ORDERS AND FAMILIES OF SEED PLANTS OF CHINA}

Copyright (C) 1993 by World Scientific Publishing Co. Pte. Ltd.

All rights reserved. This book, or parts thereof, may not be reproduced in any form or by any means, electronic or mechanical, including photocopying, recording or any information storage and retrieval system now known or to be invented, without written permission from the Publisher.

For photocopying of material in this volume, please pay a copying fee through the Copyright Clearance Center, Inc., 27 Congress Street, Salem, MA 01970, USA.

ISBN 981-02-1481-2

Printed in Singapore. 


\section{CONTENTS}

Introduction

Some Aspects of the Flora of China

xiii

List of Illustrations

The Seed Plants (Division Spermatophyta)

xvii

Gymnosperms (St

1.1 Cycadaceae

ORDER 2. GINKGOALES ….......................................................

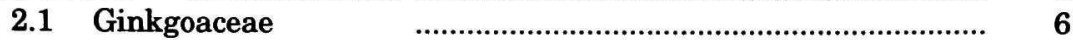

ORDER 3. CONIFERAE …....................................................

3.1 Taxaceae

3.2 Cephalotaxaceae $\quad$................................................................ 7

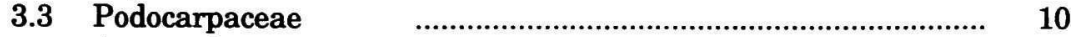

Araucariaceae

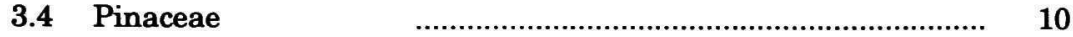

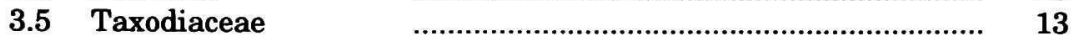

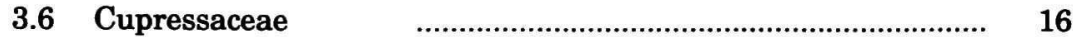

ORDER 4. GNETALES …........................................................ 16

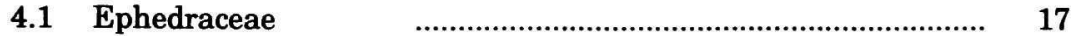

4.2 Gnetaceae

Angiosperms (Subdivision 2. Angiospermae) ................................................ 20

Dictoyledons (Class 1. Dicotyledoneae) ...................................................... 21

ORDER VERTICILLATAE …................................................... 27

Casuarinaceae $\quad$............................................................ 27

ORDER 5. PIPERALES _....................................................... 21

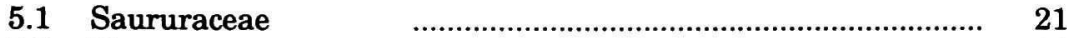

5.2 Piperaceae $\quad$......................................................... 24

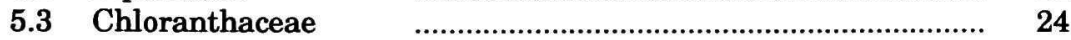

ORDER 6. SALICALES …....................................................... 24

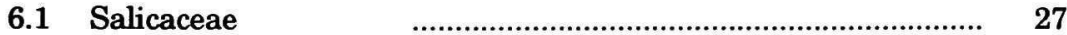

ORDER 7. MYRICALES …..................................................... 27

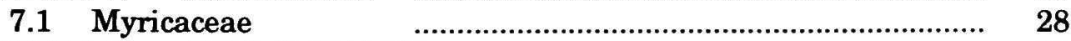

ORDER 8. JUGLANDALES........................................................... 30

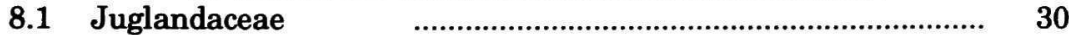

ORDER 9. FAGALES _........................................................ 30

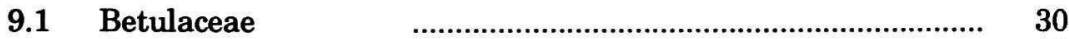

9.2 Fagaceae

ORDER 10. URTICALES _...................................................... 35

10.1 Ulmaceae

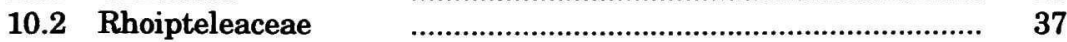

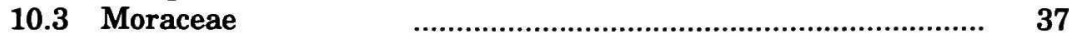

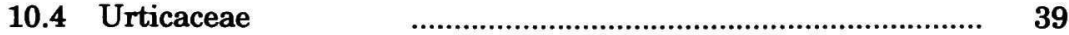

ORDER 11. PODOSTEMALES ....................................................... 41

11.1 Podostemaceae $\quad$........................................................... 41

ORDER 12. PROTEALES _............................................................ 41

12.1 Proteaceae 


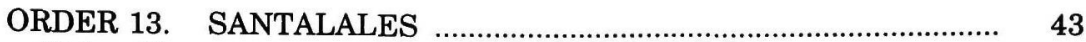

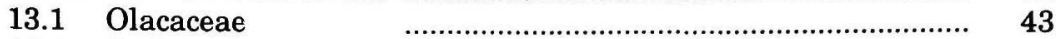

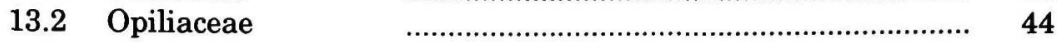

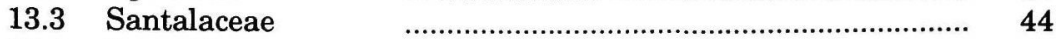

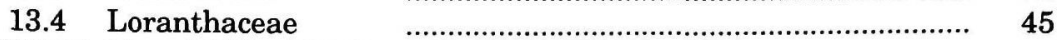

ORDER 14. ARISTOLOCHIALES ……........................................ 45

14.1 Aristolochiaceae ……................................................. 46

14.2 Rafflesiaceae ….................................................. 46

ORDER 15. BALANOPHORALES ................................................ 48

15.1 Balanophoraceae …………………............................ 48

ORDER 16. POLYGONALES ..................................................... 48

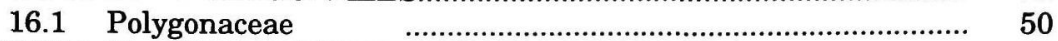

ORDER 17. CENTROSPERMAE ……........................................... 50

17.1 Chenopodiaceae …….............................................. 51

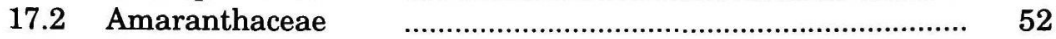

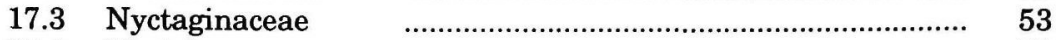

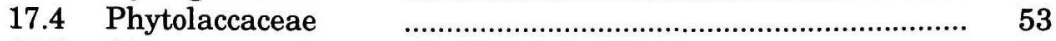

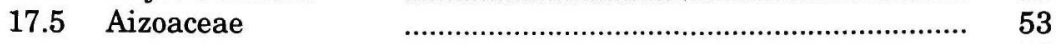

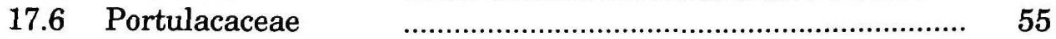

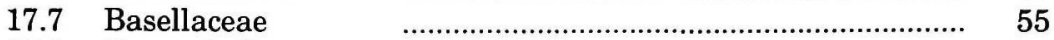

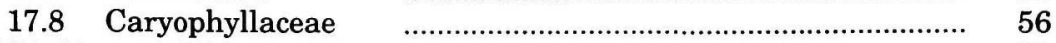

ORDER 18. RANALES …............................................... 58

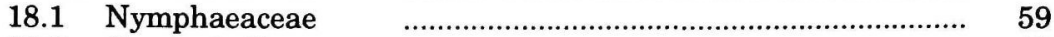

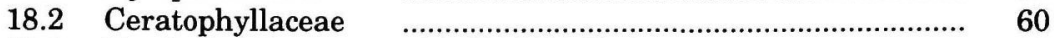

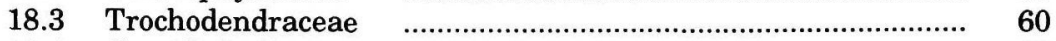

18.4 Cercidiphyllaceae $\quad$............................................................. 61

18.5 Ranunculaceae $\quad$............................................................... 61

18.6 Lardizabalaceae ……................................................. 68

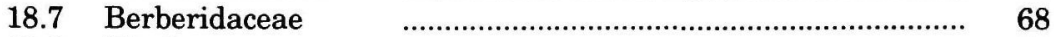

18.8 Menispermaceae …………………........................... 71

18.9 Magnoliaceae $\quad$............................................................ $\quad 72$

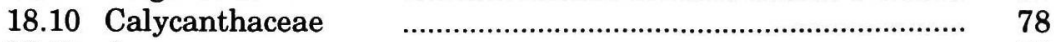

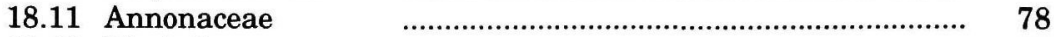

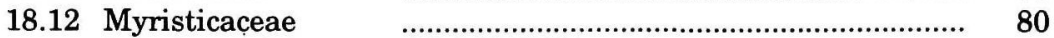

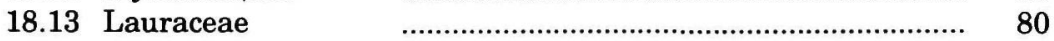

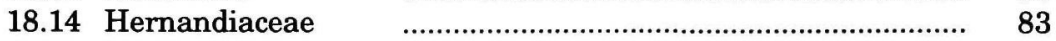

ORDER 19. RHOEADALES............................................................. 83

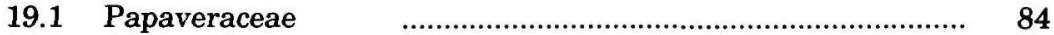

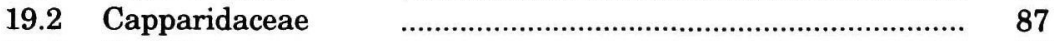

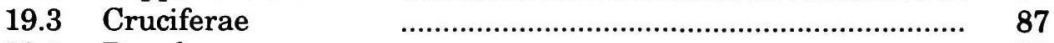

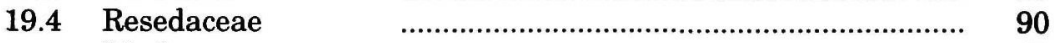

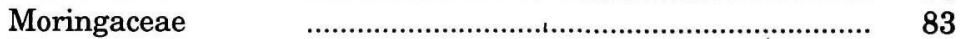

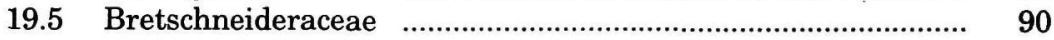

ORDER 20. SARRACENIALES …................................................ 90

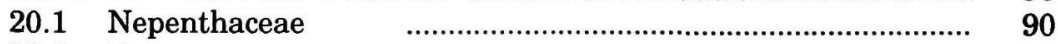

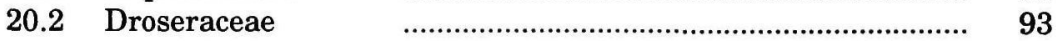


ORDER 21. ROSALES

21.1 Crassulaceae

21.2 Saxifragaceae

21.3 Pittosporaceae

21.4 Hamamelidaceae

100

21.5 Eucommiaceae

102

Platanaceae

21.6 Rosaceae

21.7 Connaraceae

21.8 Leguminosae

ORDER 22. GERANIALES

22.1 Oxalidaceae

22.2 Geraniaceae Tropaeolaceae

22.3 Linaceae 107

22.4 Erythroxylaceae

22.5 Zygophyllaceae

22.6 Rutaceae

116

119

119

22.7 Simaroubaceae

124

22.8 Burseraceae

124

22.9 Meliaceae

22.10 Malpighiaceae

22.11 Polygalaceae

22.12 Dichapetalaceae

22.13 Euphorbiaceae

22.14 Daphniphyllaceae

22.15 Callitrichaceae

ORDER 23. SAPINDALES

23.1 Buxaceae

23.2 Empetraceae

23.3 Coriariaceae

23.4 Anacardiaceae

23.5 Pentaphylacaceae

23.6 Aquifoliaceae

23.7 Celastraceae

23.8 Hippocrateaceae

23.9 Salvadoraceae

23.10 Staphyleaceae

23.11 Icacinaceae

23.12 Aceraceae

23.13 Hippocastanaceae

23.14 Sapindaceae

23.15 Sabiaceae

132

133

134

134

134

138

138

139

142

142

142

144

144

145

145

148

23.16 Balsaminaceae 
ORDER 25. MALVALES

25.1 Elaeocarpaceae

25.2 Tiliaceae

25.3 Malvaceae

25.4 Bombacaceae

25.5 Sterculiaceae

ORDER 26. PARIETALES

26.1 Dilleniaceae

26.2 Actinidiaceae

26.3 Ochnaceae

166

26.4 Theaceae

26.5 Guttiferae

168

26.6 Dipterocarpaceae

171

26.7 Elatinaceae

172

26.8 Frankeniaceae

172

26.9 Tamaricaceae

172

26.10 Cistaceae

Bixaceae

175

163

26.11 Violaceae

175

26.12 Flacourtiaceae

175

26.13 Stachyuraceae

26.14 Passifloraceae

Caricaceae

176

176

163

26.15 Datiscaceae

179

26.16 Begoniaceae

179

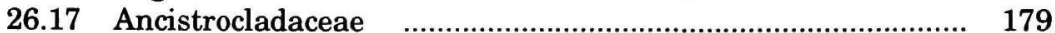

ORDER OPUNTIALES $\quad$....................................................... 163

Cactaceae

ORDER 27. MYRTIFLORAE

Thymelaeaceae

27.2 Elaeagnaceae

27.3 Lythraceae

27.4 Sonneratiaceae

186

187

27.5 Crypteroniaceae

Punicaceae

27.6 Lecythidaceae

27.7 Rhizophoraceae

27.8 Nyssaceae

27.9 Alangiaceae

27.10 Combretaceae

27.11 Myrtaceae

27.12 Melastomataceae

27.13 Trapaceae

27.14 Onagraceae 


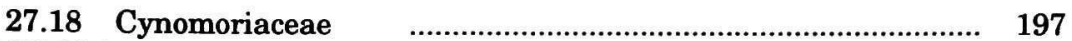

ORDER 28. UMBELLIFLORAE _.................................................. 197

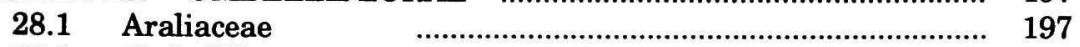

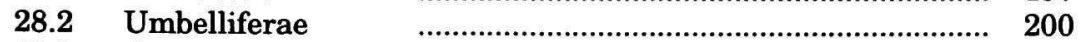

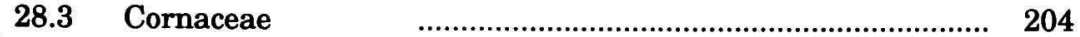

ORDER 29. DIAPENSIALES............................................................. 206

29.1 Diapensiaceae …...................................................... 206

ORDER 30. ERICALES …..................................................... 206

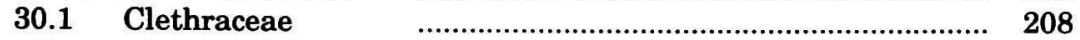

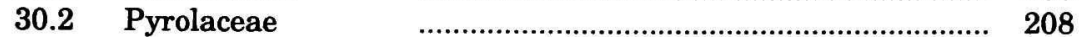

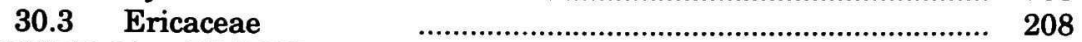

ORDER 31. PRIMULALES ................................................................ 214

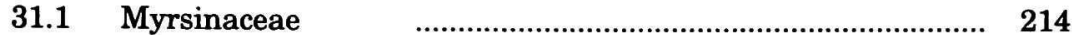

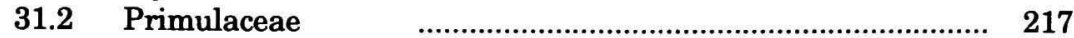

ORDER 32. PLUMBAGINALES …..................................................218

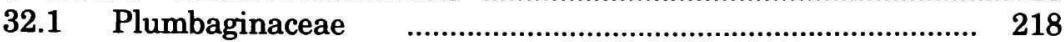

ORDER 33. EBENALES …................................................. 218

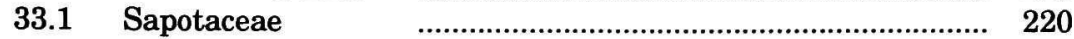

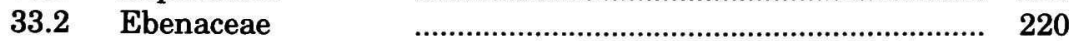

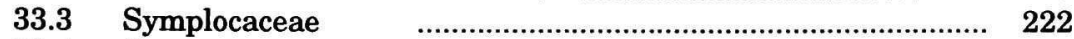

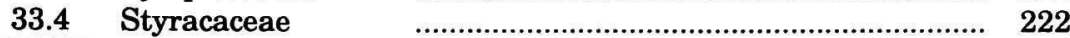

ORDER 34. CONTORTAE _................................................... $\quad 225$

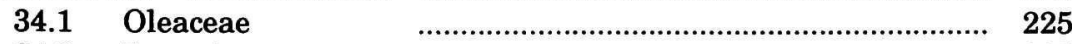

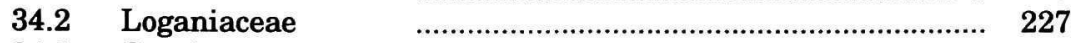

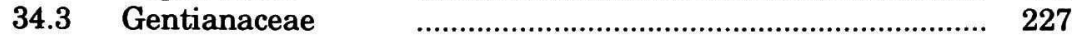

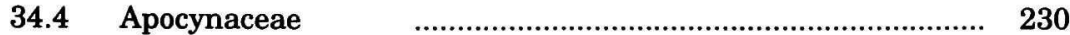

24.5 Asclepiadaceae …….................................................... 234

ORDER 35. TUBIFLORAE ............................................................ 236

35.1 Convolvulaceae …...................................................... 238

35.2 Polemoniaceae …............................................................. 240

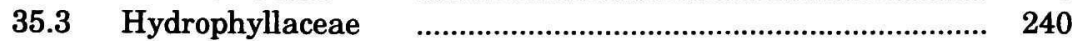

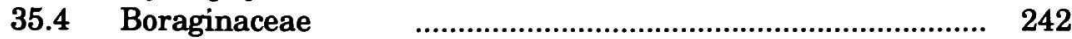

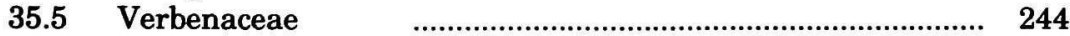

35.6 Labiatae $\quad$.......................................................... 246

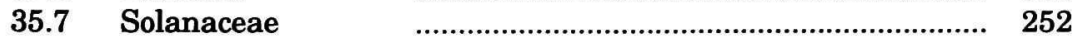

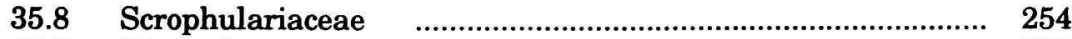

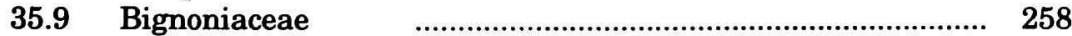

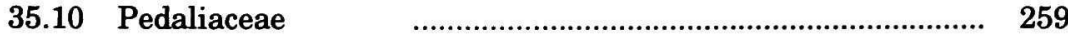

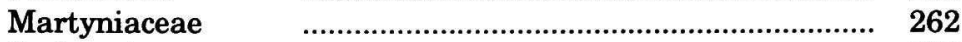

35.11 Orobanchaceae ….................................................... 262

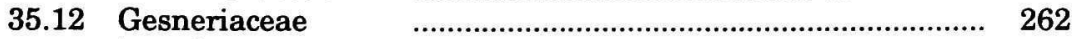

35.13 Lentibulariaceae …................................................... 266

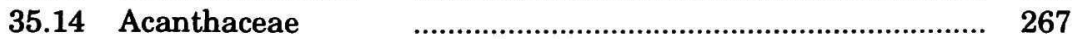

36.15 Myoporaceae …................................................... 269

35.16 Phrymataceae ….................................................. 271

ORDER 36. PLANTAGINALES …................................................ 271

36.1 Plantaginaceae ….......................................................... 271 


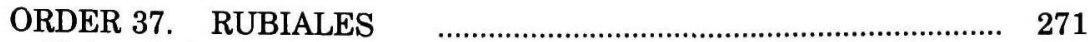

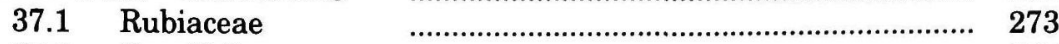

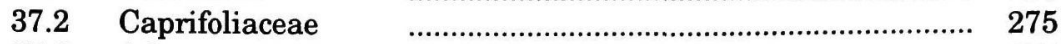

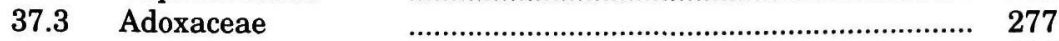

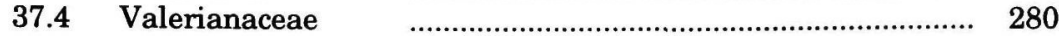

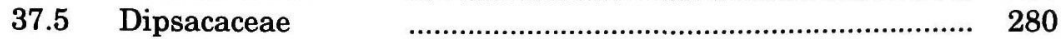

ORDER 38. CUCURBITALES......................................................... 283

38.1 Cucurbitaceae ……................................................... 283

ORDER 39. CAMPANULALES........................................................... 285

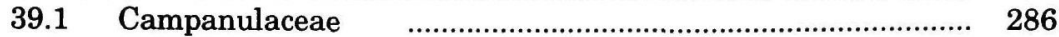

39.2 Goodeniaceae …................................................... 288

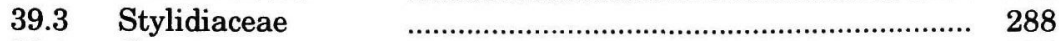

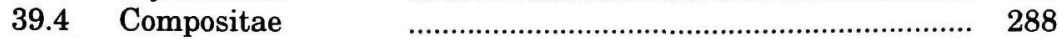

Monocotyledons (Class 2. Monocotyledoneae) ............................................ 300

ORDER 40. PANDANALES.......................................................... 300

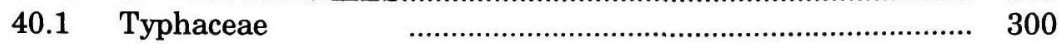

40.2 Pandanaceae ………............................................. 300

40.3 Sparganiaceae ….................................................... 303

ORDER 41. HELOBIAE _......................................................... 303

41.1 Aponogetonaceae ……………………............................... 305

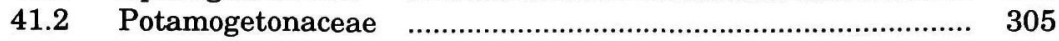

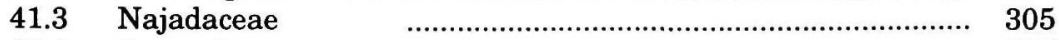

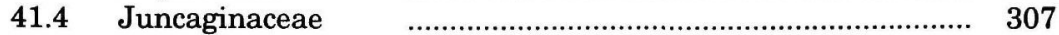

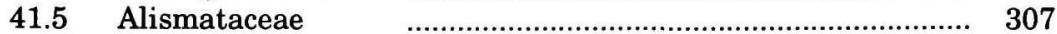

41.6 Butomaceae …............................................. 307

$41.7 \quad$ Hydrocharitaceae …….................................................. 309

ORDER 42. TRIURIDALES........................................................... 309

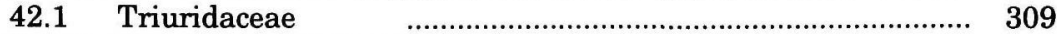

ORDER 43. GLUMIFLORAE ........................................................ 311

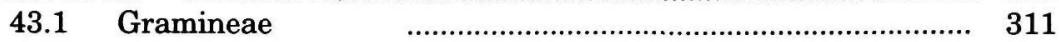

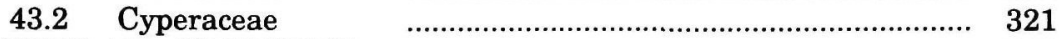

ORDER 44. PRINCIPES ….............................................. 324

44.1 Palmae

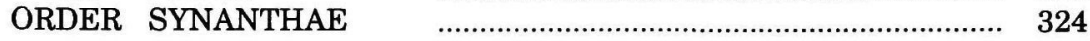

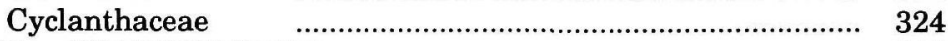

ORDER 45. SPATHIFLORAE.............................................................. 325

45.1 Araceae

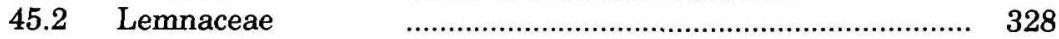

ORDER 46. FARINOSAE _................................................. $\quad 330$

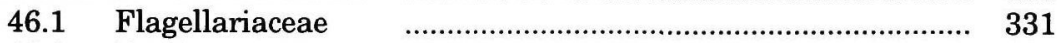

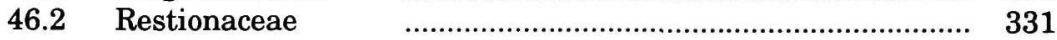

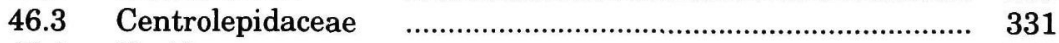

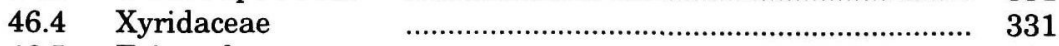

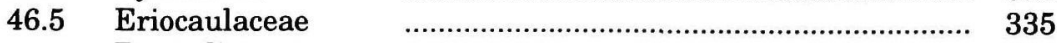

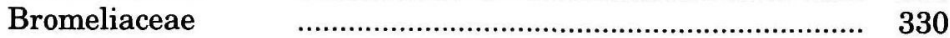

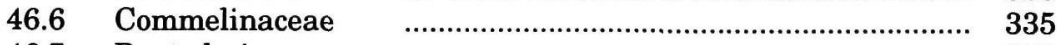

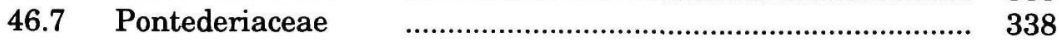




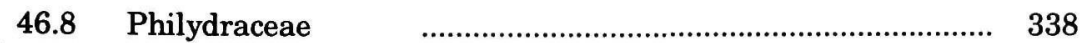

ORDER 47. LILIFLORAE _...................................................... 342

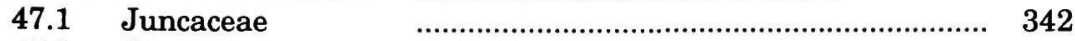

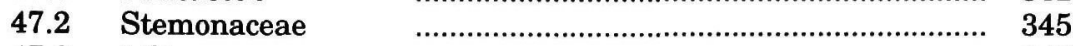

$47.3 \quad$ Liliaceae $\quad$.......................................................... 345

47.4 Amaryllidaceae …................................................... $\quad 350$

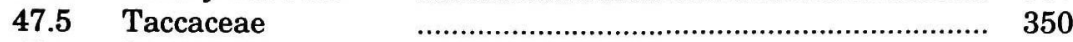

47.6 Dioscoreaceae …............................................... 353

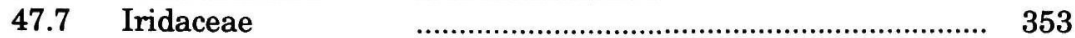

ORDER 48. SCITAMINEAE............................................................ 355

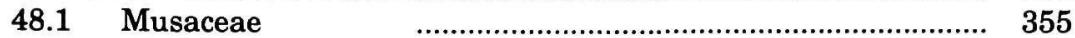

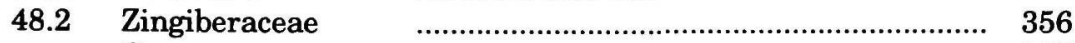

Cannaceae $\quad$................................................... 355

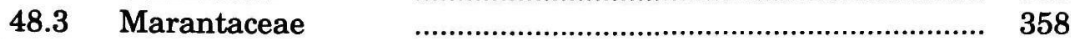

ORDER 49. MICROSPERMAE ...................................................... 358

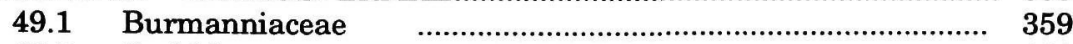

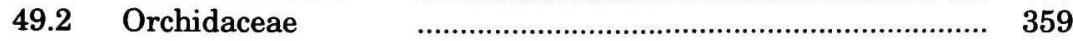

APPENDIX I. Outline of Classification of Angiosperms or Magnoliophyta, based on A. Cronquist, 1988.

367

APPENDIX II. Key to the families. .................................................... 375

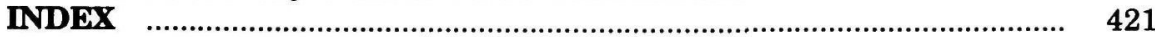


This page is intentionally left blank 


\section{INTRODUCTION}

The present volume is an enlarged and improved version of a previously published book on the seed plants of Taiwan (Keng 1987). It is an attempt to give a concise account of the orders and families of seed plants which are native to China. For the arrangement and circumscription of these orders and families, we followed closely the plan of Flora Reipublicae Popularis Sinicae (in progress) which in turn, was largely based on the 11th edition of Engler's Syllabus (1936).

Within each order, a list of the native families, a brief account of the diagnostic characters and a key to these families, if more than one, are presented. And within each family, there are also a simple description of the family and, in most cases, a synoptic key to all the native genera. Families and genera which are endemic to China, and plants of special interest or of significant importance are generally mentioned. Many major taxonomic changes in modern systems such as regrouping or transposition of some families or genera are often briefly noted.

For the keys to genera, especially those in the larger families, we followed those that appeared in Anonymous' Claves (1979). In general, these keys have been much condensed and simplified into synoptic forms and some modifications have been made. For this we are deeply indebted to the contributors and editors of the Claves, without those superbly constructed dichotomous keys, the present work would be much more difficult to compile. The reduction of a small number of genera listed in the Claves into synonyms is largely based on Willis (1973), How (1982) or Mabberley (1987).

The sequence and delimitation of orders and families of Gymnosperms are maintained with minimal changes in modern classification systems. Those of Angiosperms, however, have been significantly altered during the past few decades. For comparison, an outline of classification of Angiosperms or Magnoliophyta adapted from Cronquist's system (1988) is chosen as an example and presented in Appendix I. A lengthy key to the families of Chinese seed plants largely adapted from the Claves (1979) is presented in Appendix II at the end of this volume.

The authors wish to express their gratitude to Professor Wu Chen-yih ( 吳征鐫) for reading the entire manuscript and making numerous useful suggestions, and to Profs. Wang Fu-hsiung (王伏雄), Chen Feng-hua ( 陳封壞) and Lin Ying ( 林英 ) for their encouragement. Thanks also due to Profs. Chen

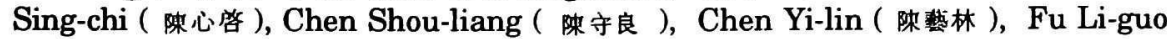
( 傅立國 ), Goh Chong-Jin ( 吳宗仁), Hew Choy-sin (丘才新), Hou Ding ( 侯定 ), Law Yuh-wu (劉玉臺), Liu Liang (劉亮 ), Lo Hsien-shui ( 罪虜瑞), Lu An-ming ( 路安民 ), Lu Ling-ti ( 陸玲姊 ), She Mong-lan ( 余孟蔽 ), Wang Wen-tsia ( 王文采 ), Yang Han-pi ( 楊漢珵 ) and many others who either read part of the manuscript or extended their help in various ways.

Illustrations of over 160 species are mostly reproduced from the 5-volumed Anonymous' Iconographia (1972-76), a few others from Flora Reipublicae 
Popularis Sinicae and Flora Yunnanica. The artists whose skilful drawings appeared in this book include, among others, Mlles. Zhang Tai-li ( 㖘泰莉) (her colour drawing of Ginkgo is reproduced on the cover of this book), Wang Jin-feng

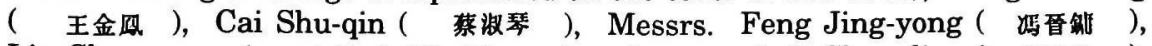

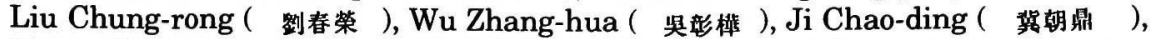
Zhang Chung-fang ( 㖘春芳 ). Madam Sally Soh typed the entire manuscript; Mrs. Ro-siu Keng went through the text and corrected numerous errors and misspellings especially amongst the generic names; finally, Ms. Jennifer Gan, the editor of World Scientific Publishing Co. offered useful technical advice and assistence. To all of them, the authors wish to express their sincere thanks.

\section{References}

Anonymous (1972-76)

Iconographia Cormophytorum Sinicorum（中國高等植物圆器 ）. 5 vols. edited by the Botanical Institute, Academia Sinica. Beijing: Science Press.

Anonymous (1979)

Claves Familiarum Generumque Cormophytorum Sinicorum（中國高等植物 科雖检索表 ) edited by the Botanical Institute, Academia Sinica. Beijing: Science Press.

Cronquist, A. (1988)

The Evolution and Classification of Flowering Plants. 2nd. ed. N.Y.: New York Botanical Garden.

Engler, A. (1936)

Syllabus der Pflanzenfamilien. 11th ed. revised by L. Diels. Berlin: Gebrueder Borntraeger.

How, F. C. (1982)

A dictionary of the families and genera of Chinese Seed Plants ( 中國種子 植物科屬詞典 ). 2nd ed. revised by T. L. Wu et al. Beijing: Science Press.

Keng, H. (1987)

Orders and Families of seed plants of Taiwan. Taipei: Council of Agriculture.

Mabberley, D. J. (1987)

The Plant Book -- A portable dictionary of the higher plants. Cambridge University Press.

Willis, J. C. (1973)

A Dictionary of the Flowering Plants and Ferns. 8th ed., revised by H. K. Airy Shaw. Cambridge University Press. 


\section{Some Aspects of the Flora of China}

1. Number of families, genera and species of Seed Plants native to China.

\begin{tabular}{|c|c|c|c|c|}
\hline & \multicolumn{2}{|c|}{ Family } & Genera & Species \\
\hline Gymnosperms & \multicolumn{2}{|c|}{10} & 34 & 192 \\
\hline $\begin{array}{l}\text { Angiosperms } \\
\text { Dicotyledons } \\
\text { Monocotyledons }\end{array}$ & $\begin{array}{r}\mathrm{E} \\
179 \\
36 \\
\end{array}$ & $\begin{array}{r}\mathrm{C} \\
205 \\
45 \\
\end{array}$ & $\begin{array}{r}2,190 \\
613 \\
\end{array}$ & $\begin{array}{r}\text { c. } 21,902 \\
\text { c. } 4,660 \\
\end{array}$ \\
\hline Total & 225 & 260 & 2,837 & c. 26,754 \\
\hline
\end{tabular}

( $\mathrm{E}=$ Engler's system, 1936. $\mathrm{C}=$ Cronquist's system, 1988)

2. Some of the larger families

a. Families with about 1,000 native species and above:

Compositae

Leguminosae

Gramineae

Orchidaceae

Rosaceae
230 genera and c. 2,800 species

130 gen. and c. $1,450 \mathrm{spp}$.

225 gen. and c. 1,300 spp.

166 gen. and c. 1,200 spp.

500 gen. and c.1,000 spp.

b. Families with about 500-900 native species:

Labiatae

Cyperaceae

Ranunculaceae

Ericaceae

Liliaceae

Scrophulariaceae

Umbelliferae

Saxifragaceae

Primulaceae
99 genera and c. 800 species.

31 gen. and c.750 spp.

40 gen. and c.720 spp.

16 gen. and c.700 spp.

54 gen. and c. $620 \mathrm{spp}$.

60 gen. and c. 600 spp.

95 gen. and c.525 spp.

28 gen. and c.500 spp.

11 gen. and c.500 spp.

3. Families endemic to China.

Ginkgoaceae

Bretschneideraceae

Eucommiaceae

4. Number of genera endemic to China.

About 145 genera (Gymnosperms 8, Dicots 122, Monocots 15) or 0.05 per cent are endemic. 
This page is intentionally left blank 


\section{List of Illustrations}

Cover

Fig. $1 \quad 1.1$

Fig. 2

2.1

Fig. 3

Fig. 4

3.1

3.2

Fig. 5

3.3

Fig. 6

Fig. 7

Fig. 8

Fig. 9

Fig. 10

Fig. 11

Fig. 12

Fig. 13

Fig. 14

Fig. 15

Fig. 16

Fig. 17

Fig. 18

Fig. 19

Fig. 20

Fig. 21

Fig. 22

Fig. 23

Fig. 24

Fig. 25

Fig. 26

Fig. 27

Fig. 28

Fig. 29

Fig. 30

Fig. 31

Fig. 32

Fig. 33

Fig. 34

Fig. 35

Fig. 36

Fig. 37

Fig. 38

Fig. 39

5.2

5.3

6.1

8.1

9.1a

9.1b

9.2

10.1

10.2

10.3

14.1

15.1

17.4

17.8

18.4
Ginkgo biloba L. (by Ms Zhang Tai-li)

Cycas revoluta Thunb. (1-6) and C. szechuanensis Cheng et L. K. Fu (7-8) (Cycadaceae)

Ginkgo biloba L. (Ginkgoaceae)

Amentotaxus yunnanensis Li (Taxaceae)

Cephalotaxus hainanensis $\mathrm{Li}$ (1) and $C$. sinensis $\mathrm{Li}$ (2) (Cephalotaxaceae)

Podocarpus nagi Zoll. et Mor. (Podocarpaceae)

Cathaya argyrophylla Chun et Kuang (Pinaceae)

Metasequoia glyptostroboides $\mathrm{Hu}$ et Cheng (Taxodiaceae)

Juniperus chinensis L. (Cupressaceae)

Ephedra sinica Stapf. (Ephedraceae)

Gnetum cleistostachyum C. Y. Cheng (1-3) and G. pendulum C. Y. Cheng (4-5) (Gnetaceae)

Houttuynia cordata Thunb. (Saururaceae)

10.4

$18.3 \mathrm{a}$

Piper hainanense Hemsl. (Piperaceae)

Chloranthus spicatus Makino (Chloranthaceae)

Populus alba L. (Salicaceae)

Juglans cathayensis Dode (Juglandaceae)

Betula albo-sinensis Burck. (Betulaceae)

Corylus heterophylla Fisch. ex Bess. (Betulaceae)

Fagus longipetiolata Seem. (Fagaceae)

Ulmus parvifolia Jacq. (Ulmaceae)

Rhoiptelea chiliantha Diels et Hand. -Mazz. (Rhoipteleaceae)

Humulus scandens Merr. (Moraceae)

Boehmeria nivea Gaud. (Urticaceae)

Aristolochia debilis Sieb. et Zucc. (Aristolochiaceae)

Balanophora japonica Makino (Balanophoraceae)

Phytolacca acinosa Roxb. (Phytolaccaceae)

Dianthus chinensis L. (Caryophyllaceae)

Euptelea pleiospermum Hook. f. et Thoms. (Trochodendraceae)

$18.3 \mathrm{~b}$

Trochodendron aralioides Sieb. et Zucc. (Trochodendraceae)

Cercidiphyllum japonicum Sieb. et Zucc. var. sinense Rehd. et Wils. (Cercidiphyllaceae)

$18.5 \mathrm{a}$

$18.5 \mathrm{~b}$

18.6

18.7

$18.9 \mathrm{a}$

Kingdonia uniflora Balf. f. et W. W. Sm. (Ranunculaceae)

Paeonia papaveracea Andr. (Ranunculaceae)

Sargentodoxa cuneata (Oliv.) Rehd. et Wils. (Lardizabalaceae)

Dysosma versipellis (Hance) M. Cheng (Berberidaceae)

$18.9 \mathrm{~b}$

$18.9 \mathrm{c}$

Liriodendron chinense (Hemsl.) Sarg. (Magnoliaceae)

Magnolia officinalis Rehd. et Wils. (Magnoliaceae)

Tetracentron sinense Oliv. (Magnoliaceae)

18.10

Calycanthus chinensis Cheng et (Calycanthaceae)

18.11 Mitrephora thorelii Pierre (Annonaceae)

18.13

Sassafras tzumu Hemsl. (Lauraceae) 
Fig. 40

Fig. 41

Fig. 42

Fig. 43

Fig. 44

Fig. 45

Fig. 46

Fig. 47

Fig. 48

Fig. 49

Fig. 50

Fig. 51

Fig. 52

Fig. 53

Fig. 54

Fig. 55

Fig. 56

Fig. 57

Fig. 58

Fig. 59

Fig. 60

Fig. 61

Fig. 62

Fig. 63

Fig. 64

Fig. 65

Fig. 66

Fig. 67

Fig. 68

Fig. 69

Fig. 70

Fig. 71

Fig. 72

Fig. 73

Fig. 74

Fig. 75

Fig. 76

Fig. 77

Fig. 78

Fig. 79

Fig. 80

Fig. 81

Fig. 82

Fig. 83

Fig. 84

Fig. 85

Fig. 86 19.1a

$19.1 \mathrm{~b}$

19.4

19.5

20.1

21.2a

21.2b

21.4

21.5

21.6

21.8a

$21.8 \mathrm{~b}$

22.2

22.5

$22.6 \mathrm{a}$

$22.6 \mathrm{~b}$

22.7

22.13

23.1

23.3

23.4

23.6

23.7

23.10

23.12

23.13

23.14

23.15

23.16

24.2

25.1

25.2

25.3

25.5

26.1

26.2

26.4

26.5

26.6

26.9

26.12

26.13

26.15

26.16

27.1

27.2

27.4
Corydalis yanhusuo W. T. Wang (Papaveraceae)

Papaver nudicaule L. (Papaveraceae)

Oligomeris linifolia Machride (Resedaceae)

Bretschneidera sinensis Hemsl. (Bretschneideraceae)

Nepenthes mirabilis Druce (Nepenthaceae)

Deutzia grandiflora Bge. (Saxifragaceae)

Oresitrophe rupifraga Bge. (Saxifragaceae)

Corylopsis sinensis Hemsl. (Hamamelidaceae)

Eucommia ulmoides Oliv. (Eucommiaceae)

Crataegus pinnatifida Bge. (Rosaceae)

Caesalpinia sepiaria Roxb. (Leguminosae)

Astragalus mongholicus Bge. (Leguminosae)

Geranium delavayi Franch. (Geraniaceae)

Peganum nigellastrum Bge. (Zygophyllaceae)

Zanthoxylum planispinum Sieb. et Zucc. (Rutaceae)

Phellodendron amurense Rupr. (Rutaceae)

Ailanthus altissima Swingle (Simaroubaceae)

Euphorbia helioscopia L. (Euphorbiaceae)

Buxus microphylla Sieb. et Zucc. var. sinica Rehd. et Wils.

(Buxaceae)

Coriaria sinica Maxim. (Coriariaceae)

Rhus verniciflua Stokes (Anacardiaceae)

Ilex chinensis Sims (Aquifoliaceae)

Celastrus paniculatus Willd. (Celastraceae)

Tapiscia sinensis Oliv. (Staphyleaceae)

Acer oliverianum Pax (Aceraceae)

Aesculus wilsonii Rehd. (Hippocastanaceae)

Koelreuteria paniculata Laxm. (Sapindaceae)

Meliosma cuneifolia Franch. (Sabiaceae)

Impatiens furcillata Hemsl. (Balsaminaceae)

Vitis amurensis Rupr. (Vitaceae)

Elaeocarpus decipiens Hemsl. (Elaeocarpaceae)

Tilia henryana Szyszyl. var. subglabra V. Engl. (Tiliaceae)

Abelmoschus manihot Medic. (Malvaceae)

Heritiera littoralis Dryand. (Sterculiaceae)

Dillenia turbinata Finet et Gagnep. (Dilleniaceae)

Actinidia latifolia Merr. (Actinidiaceae)

Camellia hongkongensis Seem. (Theaceae)

Hypericum delavayi Franch. (Guttiferae)

Hopea hainanensis Merr. et Chun (Dipterocarpaceae)

Tamarix juniperina Bge. (Tamaricaceae)

Idesia polycarpa Maxim. (Flacourtiaceae)

Stachyurus yunnanensis Franch. (Stachyuraceae)

Tetrameles nudiflora $\mathrm{R}$. Br. (Datiscaceae)

Begonia pedatifida Levl. (Begoniaceae)

Daphne tangutica Maxim. (Thymelaeaceae)

Elaeagnus lanceolata Warb. (Elaeagnaceae)

Sonneratia caseolaris Engl. (Sonneratiaceae) 
Fig. 87

Fig. 88

Fig. 89

Fig. 90

Fig. 91

Fig. 92

Fig. 93

Fig. 94

Fig. 95

Fig. 96

Fig. 97

Fig. 98

Fig. 99

Fig. 100

Fig. 101

Fig. 102

Fig. 103

Fig. 104

Fig. 105

Fig. 106

Fig. 107

Fig. 108

Fig. 109

Fig. 110

Fig. 111

Fig. 112

Fig. 113

Fig. 114

Fig. 115

Fig. 116

Fig. 117

Fig. 118

Fig. 119

Fig. 120

Fig. 121

Fig. 122

Fig. 123

Fig. 124

Fig. 125

Fig. 126

Fig. 127

Fig. 128

Fig. 129

Fig. 130

Fig. 131
27.8

27.12

27.14

28.1

28.2

28.3

29.1

30.1

30.2

$30.3 \mathrm{a}$

$30.3 \mathrm{~b}$

31.1

31.2

32.1

33.2

$33.4 \mathrm{a}$

$33.4 \mathrm{~b}$

34.1

34.2

$34.3 \mathrm{a}$

$34.3 \mathrm{~b}$

35.1

35.2

35.4

35.6

35.7

$35.8 \mathrm{a}$

$35.8 \mathrm{~b}$

35.9

35.10

35.11

35.12

35.14

35.15

36.1

$37.2 \mathrm{a}$

$37.2 \mathrm{~b}$

37.3

37.4

37.5

38.1

39.1

$39.4 \mathrm{a}$

$39.4 \mathrm{~b}$

40.1
Davidia involucrata Baill. (Nyssaceae)

Melastoma dodecandrum Lour. (Melastomataceae)

Epilobium amurense Hausskn. (Onagraceae)

Panax schinseng Nees. (Araliaceae)

Daucus carota L. (Umbelliferae)

Cornus macrophylla Wall. (Cornaceae)

Shortia sinensis Hemsl. (Diapensiaceae)

Clethra cavaleriei Levl. (Clethraceae)

Cheilotheca humilis (D. Don) H. Keng (Monotropastrum macrocarpus $\mathrm{H}$. Andres) (Pyrolaceae)

Rhododendron taggianum Hutch. (Ericaceae)

Vaccinium retusum Hook. f. (Ericaceae)

Ardisia virens Kurz (Myrsinaceae)

Trientalis europaea L. (Primulaceae)

Ceratostigma griffithii Clarke (Plumbaginaceae)

Diospyros diversilimba Merr. et Chun (Ebenaceae)

Melliodendron xylocarpum Hand. -Mazz. (Styracaceae)

Parastyrax macrophylla C. Y. Wu et K. M. Feng (Styracaceae)

Syringa sweginzowii Kohne et Lingelsh (Oleaceae)

Buddleja davidii Franch. (Loganiaceae)

Gentiana davidii Franch. (Gentianaceae)

Nymphoides indica O. Ktze. (Gentianaceae)

Calystegia sepium R. Br. (Convolvulaceae)

Polemonium laxiflorum Kitam. (Polemoniaceae)

Bothriospermum chinense Bge. (Boraginaceae)

Lamium barbatum Sieb. et Zucc. (Labiatae)

Leucophysalis sinicum (Kuang et Lu) Averett (Physaliastrum sinicum Kuang et Lu) (Solanaceae)

Paulownia tomentosa (Thunb.) Steud. (Scrophulariaceae)

Pedicularis decora Franch. (Scrophulariaceae)

Catalpa bungei C. A. Mey. (Bignoniaceae)

Sesamum indicum L. (Pedaliaceae)

Boschniakia rossica Fedtsch. et Flerov. (Orobanchaceae)

Didymocarpus stenanthos Clarke (Gesneriaceae)

Pararuellia delavayana (Baill.) E. Hossain (Acanthaceae)

Myoporum bontioides A. Gray (Myoporaceae)

Plantago major L. (Plantaginaceae)

Kolkwitzia amabilis Graebn. (Caprifoliaceae)

Lonicera hypoglauca Miq. (Caprifoliaceae)

Adoxa moschatellina L. (Adoxaceae)

Patrinia angustifolia Hemsl. (Valerianaceae)

Dipsacus japonicus Miq. (Dipsacaceae)

Trichosanthes kirilowii Maxim. (Cucurbitaceae)

Adenophora wawreana A. Zahlbr. (Campanulaceae)

Crepis crocea Babcock (Compositae)

Chrysanthemum indicum L. (Compositae)

Typha orientalis Presl (Typhaceae) 
Fig. 132

Fig. 133

Fig. 134

Fig. 135

Fig. 136

Fig. 137

Fig. 138

Fig. 139

Fig. 140

Fig. 141

Fig. 142

Fig. 143

Fig. 144

Fig. 145

Fig. 146

Fig. 147

Fig. 148

Fig. 149

Fig. 150

Fig. 151

Fig. 152

Fig. 153

Fig. 154

Fig. 155

Fig. 156

Fig. 157

Fig. 158

Fig. 159

Fig. 160

Fig. 161

Fig. 162

Fig. 163
40.2

40.3

41.2

41.5

41.7

43.1a

43.1b

43.1c

43.2

45.1a

45.1b

45.2

46.1

46.2

46.3

46.4

46.5

46.4

46.7

46.8

47.1

47.2

47.3a

$47.3 \mathrm{~b}$

47.4

47.5

47.7

48.2

49.1

$49.2 \mathrm{a}$

$49.2 \mathrm{~b}$

$49.2 \mathrm{c}$
Pandanus forceps Mart. (Pandanaceae)

Sparganium simplex Huds. (Sparganiaceae)

Potamogeton distinctus A. Benn. (Potamogetonaceae)

Alisma orientalis Juzep. (Alismataceae)

Ottelia esquirolii Dandy (Hydrocharitaceae)

Bambusa sinospinosa Maclure (Gramineae)

Festuca modesta Stued. (Gramineae)

Hymenachne pseudointerrupta C. Muell. (Gramineae)

Carex stipata Muhlenb. (Cyperaceae)

Acorus calamus L. (Araceae)

Pinellia ternata (Thunb.) Breitenb. (Araceae)

Spirodela polyrrhiza (L.) Schleid. (Lemnaceae)

Flagellaria indica L. (Flagellariaceae)

Leptocarpus disjunctus Mast. (Restionaceae)

Centrolepis banksii (R. Br.) R. et S. (Centrolepidaceae)

Xyris indica L. (Xyridaceae)

Eriocaulon truncatum Ham. (Eriocaulaceae)

Murdannia keisak (Hassk.) Hand.-Mazz. (Commelinaceae)

Monochoria korsakowii Regel et Maack (Pontederiaceae)

Philydrum lanuginosum Banks (Philydraceae)

Juncus elatus Franch. et Sav. (Juncaceae)

Stemona tuberosa Lour. (Stemonaceae)

Lilium brownii F. E. Br. var. viridulum Baker (Liliaceae)

Veratrum nigrum L. (Liliaceae)

Lycoris aurea Herb. (Amaryllidaceae)

Tacca chantrieri Andre (Taccaceae)

Belamcanda chinensis (L.) DC. (Iridaceae)

Zingiber officinale Rosc. (Zingiberaceae)

Burmannia disticha L. (Burmanniaceae)

Apostasia odorata Bl. (Orchidaceae)

Paphiopedilum appletonianum Pfitzer (Orchidaceae)

Dendrobium hancockii Rolfe (Orchidaceae) 